\title{
A DRIVING SIMULATOR STUDY TO EXAMINE THE ROLE OF VEHICLE ACOUSTICS ON DRIVERS' SPEED PERCEPTION
}

\author{
Natasha Merat \& Hamish Jamson \\ Institute for Transport Studies \\ University of Leeds, UK \\ Email: n.merat@its.leeds.ac.uk
}

\begin{abstract}
Summary: In recent years, there has been a desire by vehicle manufacturers to reduce the in-cab noise of vehicles, in order to improve driver comfort and enhance the enjoyment of in-vehicle entertainment systems. This reduction of incab noise is accompanied by policy initiatives to reduce transport related noise by implementing low noise road surfaces. However, it is not known how such reductions in the availability of auditory cues affect drivers' ability to judge speed, and there is a danger that drivers will increase their speed, to compensate for the absence of auditory cues. In this study, drivers were required to maintain speed at 30 and $70 \mathrm{mph}$, in the absence of a speedometer, with and without accompanying vehicle noise. Results showed that drivers' ability to maintain the correct speed profile was much more variable in the absence of accompanying vehicle noise and this variation was found to be higher when drivers were asked to travel at higher speeds of $70 \mathrm{mph}$. Drivers were also found to travel faster than the required speed in the absence of vehicle noise, although their ability to maintain speed was generally worse at $70 \mathrm{mph}$, even in the presence of auditory cues.
\end{abstract}

\section{INTRODUCTION}

Environmental noise induced by traffic is known to have a serious impact on our health, contributing to stress, annoyance and hearing loss. Indeed, according to a report by the World Health Organisation, between 1 and $2 \%$ of the total world disease burden is thought to be attributable to traffic noise, which can be a major cause of sleep deprivation, raised blood pressure and heart disease (WHO, 2000). It is not surprising then that substantial efforts have been made in recent years to reduce traffic related noise through measures such as infrastructure improvement. Examples include investing in low height barriers and introducing low noise road surfaces. In parallel to such implementations, there have also been considerable advances in vehicle engine technologies, with "quietness" one of the major selling points of high end and luxury cars. This is chiefly because vehicle manufacturers are eager to increase driver comfort and pleasure, by creating vehicle cabs which convey little or no noise, thus allowing better use and enjoyment of in-car entertainment and communication systems.

The psychophysics of speed and motion perception is known to be determined by a combination of auditory, visual and audiovisual cues (e.g. Kemeny, \& Panerai, 2003). The role of vision and the influence of optic flow in speed perception were first outlined by Gibson (1950). Since then, many studies have highlighted the significant role of vision in driving performance and driving safety (e.g. Manser \& Hancock, 2007; see also Owsley \& McGwin, 2010 for a review). Indeed, an analysis by Sivak (1996), of articles available on PscyINFOC found that $84 \%$ of articles 
published on the theme of "driving and the senses" were related to studies on the influence of vision in driving, with sound, touch, kinaesthetics, smell and taste featured in the remaining $16 \%$ of articles.

However, it is now widely agreed that our perception of the world and its surroundings is heavily influenced by the multisensory integration of information and that our perception of information in one sensory modality can be profoundly influenced by another modality. Perhaps the most famous example here is the McGurk effect where seeing the movement of a speaker's lips influences how speech is heard (McGurk \& McDonald, 1976). Although the effect of audition on vision is perhaps not as widely investigated, striking examples include an experiment by Shams et al. (2005) where participants reported seeing multiple flashing objects on a screen, when in fact only one object was flashed, but accompanied by multiple beeps (Shams, Kamitani \& Shimojo, 2005). Sound has also been shown to alter the visual perception of object motion (Sekuler, Sekuler \& Lau, 1997; Hidaka et al., 2009). Therefore, the role of auditory feedback in driver performance and speed/motion perception cannot be ignored.

Conventionally, the noise experienced by drivers in a moving vehicle is a combination of the noise generated by the contact of the tyres with the road (rolling), and the noise from the engine plus wind turbulence related noise. Typically, in-vehicle noise levels are shown to increase with the speed of travel, as illustrated in Figure 1. Therefore, it can be argued that a reduction in auditory feedback to the driver, a feature of modern cars and road conditions, can have a detrimental effect on drivers' speed perception. As sound is shown to influence visual motion perception (e.g. Hidaka et al., 2009) its attenuation or absence in the car may lead to driver confusion, encouraging drivers to drive faster in order to compensate for the absence of the auditory cues needed for speed perception. Such undesirable increases in driving speed, leading to reduced driver safety may well be intensified by future initiatives to utilise alternate fuel technologies, in lighter, less well shielded vehicles, that present drivers with unfamiliar acoustic environments.

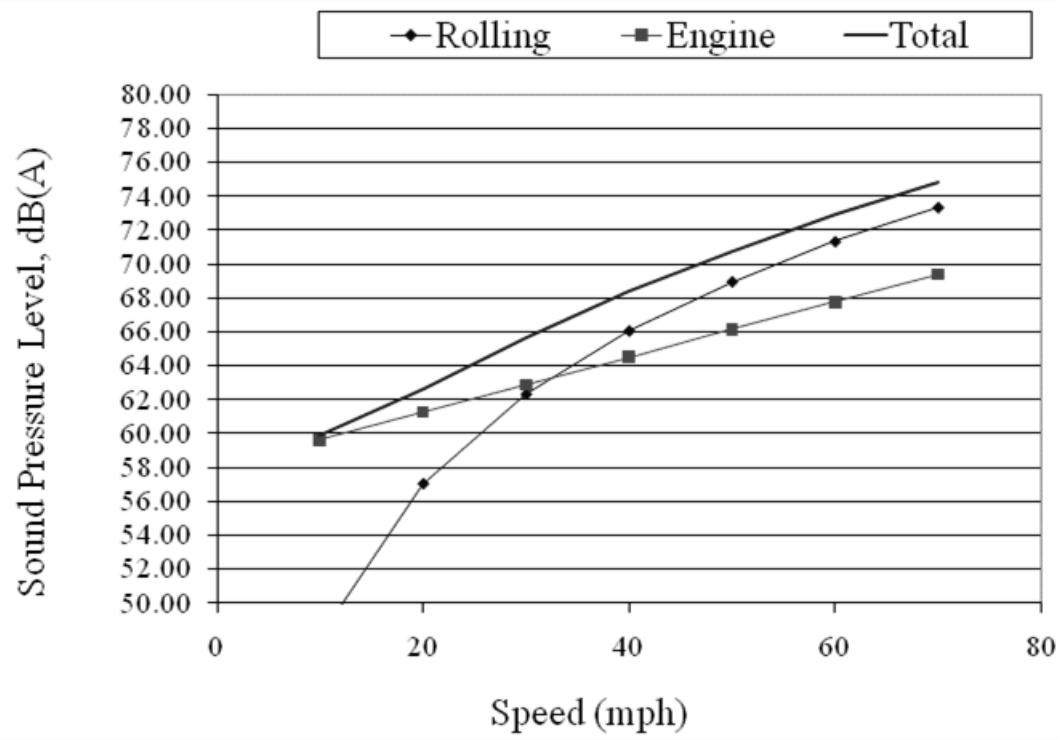

Figure 1. Sound pressure level produced by vehicle and engine noise at varying speeds of travel (EU Imagine Project, 2005) 
Only a handful of studies have investigated the effect of sound on speed perception and driving performance, and findings from these studies are conflicting. Examples include an experiment by Evans (1970) who reported an underestimation of speed by drivers asked to judge their speed of travel whilst wearing earmuffs, whilst McLane and Wierville (1975) failed to show a significant difference in driving speed judgements during a driving simulator study which compared driving with and without auditory feedback. Likewise, changes in accompanying engine sound was not seen to have an effect on participants' estimation of travelling speed, when they observed video clips of travelling cars, recorded from the drivers' viewpoint (Horswill and McKenna, 1999). Horswill and Plooy (2008) argue that to achieve accurate results in such studies, participants must be asked to report on relative judgements of speed, whereas most previous studies have used absolute judgements of speed, when assessing the effect of auditory cues on speed perception in driving. Indeed, Horswill and Plooy (2008) used a 2-alternate forced-choice methodology by presenting pairs of video clips containing recordings of cars travelling at various speeds, and asked participants to judge whether driving in scene one was faster or slower than that of scene two. Participants reported slower speeds for clips which were accompanied by sounds $5 \mathrm{~dB}$ lower than the "normal" in-car sound.

The study reported here used a combined methodology, by allowing drivers to make both absolute and relative judgements of their speed of travel, in a driving simulator experiment. Drivers were first asked to drive the simulator at two designated speeds, with access to the speedometer and accompanied by normal in-car acoustics. They were then asked to judge their speed of travel in the absence of the speedometer and their accuracy in speed perception was compared with and without normal in-cab acoustics.

\section{METHOD}

\section{Participants}

Twelve volunteers (10 male, 2 female) were recruited for this study. Drivers ranged in age from 19 to 30 years $($ Mean $=23.58, \mathrm{SD}=3.78)$. All drivers held a driving licence, with an average driving experience of 5.63 years $(\mathrm{SD}=2.3)$. All participants were briefed about the experiment and completed a consent form before commencing the drive.

\section{Design and Procedure}

The motion based University of Leeds Driving Simulator (UoLDS) was used to conduct this study (Figure 2). A within participants design was used, whereby all participants were asked to drive a 3-lane section of motorway, which did not contain any other traffic. An absence of traffic was implemented in this study because it was envisaged that any interactions with other traffic may have prevented drivers from maintaining a set speed for the required time durations (a criterion of the experiment).

A vehicle identical to that of used in the driving simulator was used to make recordings of engine and overall vehicle noise, on a real road during dry weather conditions. The graph in Figure 1 was then used to calibrate the noise produce by the simulator, presenting the correct vehicle noise heard at various speeds of travel. 

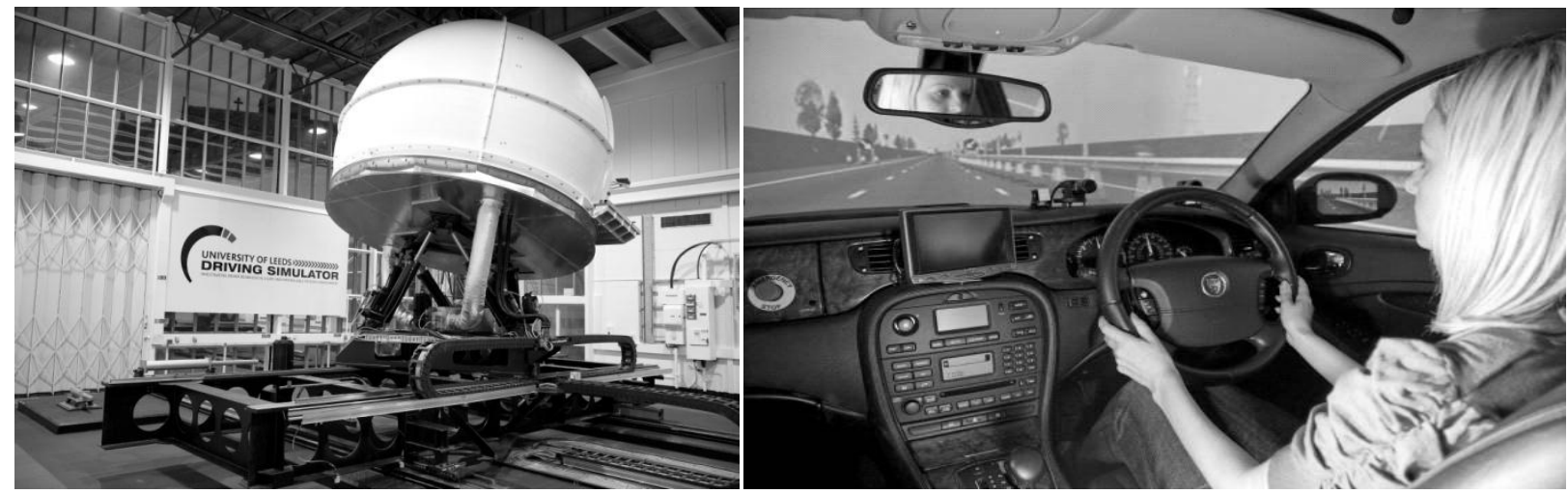

Figure 2. Exterior view (left) and vehicle cab (right) of the University of Leeds Driving Simulator

All drivers began the experiment with a 10 minute practice drive, followed by a 30 minute experimental drive divided into three sections (A, B and C). For each experimental section, participants were required to accelerate from 0 to $30 \mathrm{mph}$ and maintain their speed at $30 \mathrm{mph}$ for 2 minutes, after which they were asked to accelerate from 30 to $70 \mathrm{mph}$ and maintain this speed for 2 minutes. For the last part of each section, drivers were required to decelerate from 70 to 30 $\mathrm{mph}$ and once they reached $30 \mathrm{mph}$, maintain this speed for a further 2 minutes. After maintaining the speed at $30 \mathrm{mph}$ for a second time, participants were instructed to decelerate and bring the vehicle to a complete stop, before starting the same cycle for the next section.

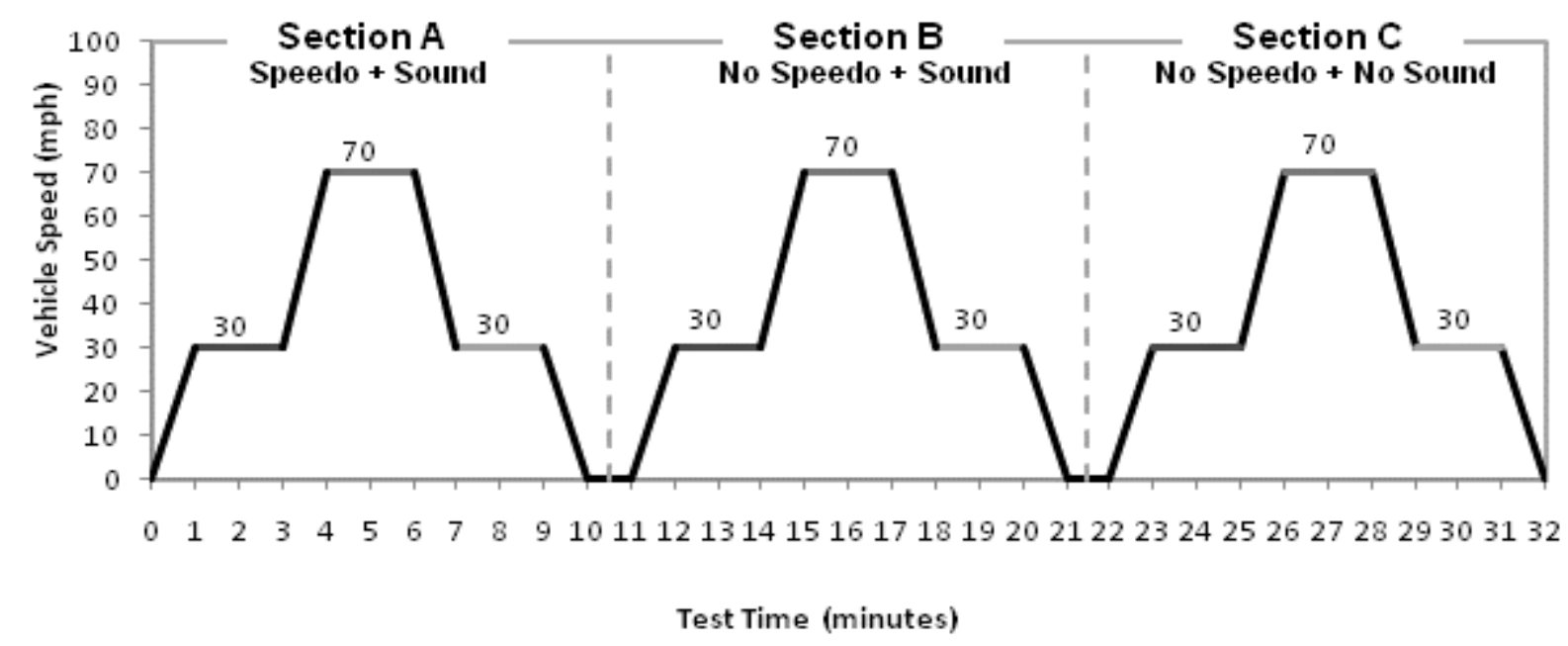

Figure 3. The ideal speed profile required from drivers in each of the three sections

The instructions to accelerate and decelerate were provided from the control room, via a two way radio. For each speed profile, participants pressed a button on the steering wheel to indicate they had achieved the desired speed requested. They then heard an auditory signal two minutes after this button was pressed, which indicated the end of that particular speed profile (see Figure 3).

To test whether drivers' speed perception is influenced by the presence/absence of engine/ rolling noise, the simulator speedometer was deactivated for sections $\mathrm{B}$ and $\mathrm{C}$ and whilst the normal acoustics of the simulator remained on for section B of the drive, they were switched off 
for section C. All drivers drove section A first, which was used to collect baseline speed measurements, as well as familiarising drivers with the different speed profiles for relative judgement purposes in sections $\mathrm{B}$ and $\mathrm{C}$. In order to reduce learning effects, after driving section A, six drivers drove section $\mathrm{B}$ followed by section $\mathrm{C}$, whilst the other six drivers drove section $\mathrm{C}$ followed by section B.

\section{RESULTS}

The average travelling speed of each of the twelve participants is plotted below (Figure 4). Perhaps not surprisingly, all drivers were able to maintain a relatively accurate speed profile when both speedometer and sound were available in Section A of the drive. However, drivers' speed perception was found to be much more variable in the absence of the speedometer and accuracy deteriorated further when drivers were asked to maintain speed in the absence of any auditory cues. As seen in Figure 4, the standard deviation of speed was also seen to be generally higher in Sections B and C, compared to section A.

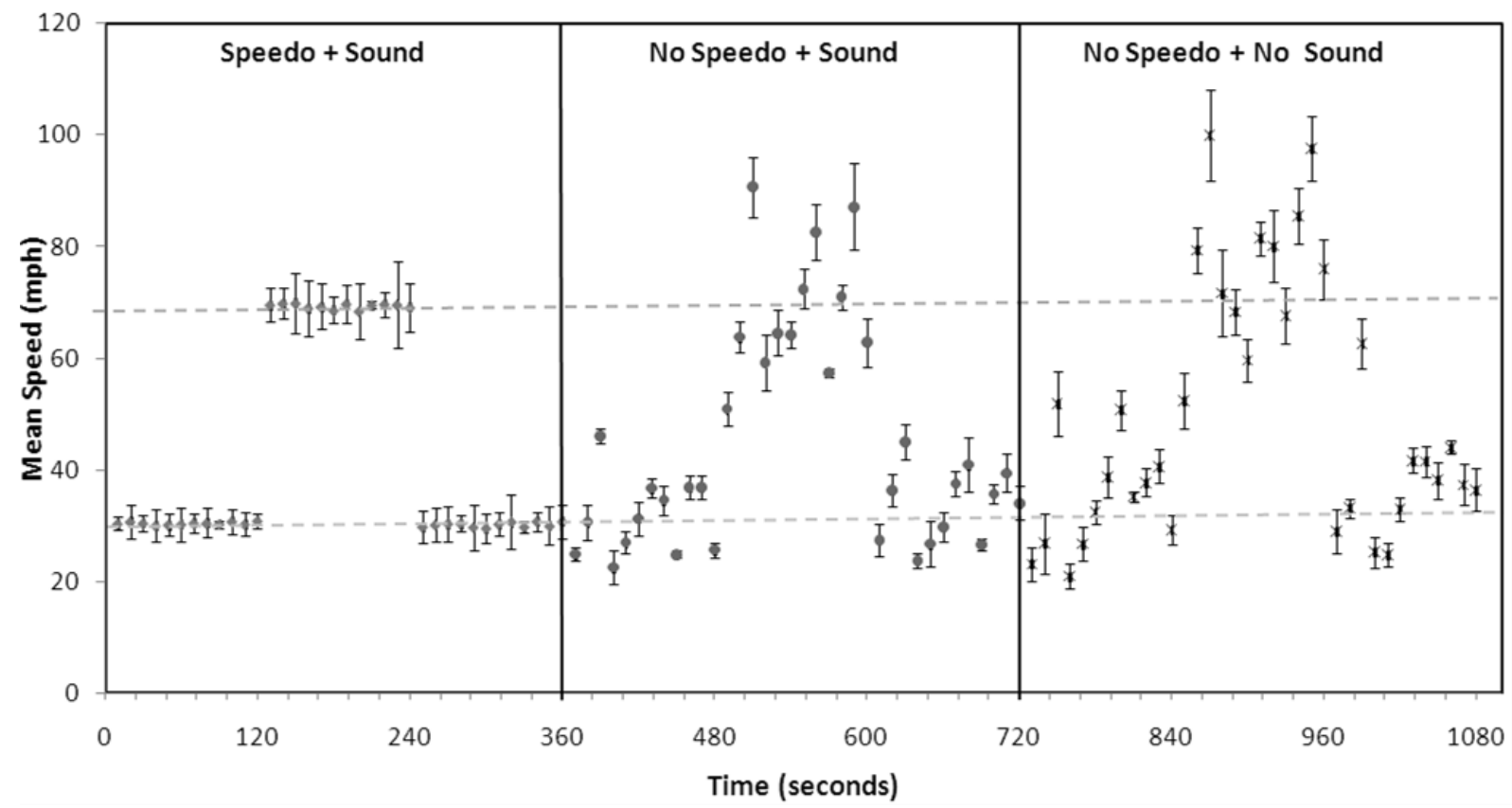

Figure 4. Mean speed of travel for each of the three sections (error bars are SD)

A $3 \times 3$ repeated measures Analysis of Variance (ANOVA) was conducted on this standard deviation of speed, comparing the value in the 3 sections (A, B and C) and at the three speed profiles (first $30 \mathrm{mph}, 2^{\text {nd }} 30 \mathrm{mph}$ and $70 \mathrm{mph}$ ). Results showed a significant main effect of section $\left(\mathrm{F}(2,22)=9.56, \mathrm{p}<.001, \eta^{2}=.465\right)$ and pair wise Bonferroni comparisons showed more speed variation in section $\mathrm{C}(\mathrm{p}<.05)$, compared to sections $\mathrm{A}$ and $\mathrm{B}$. 


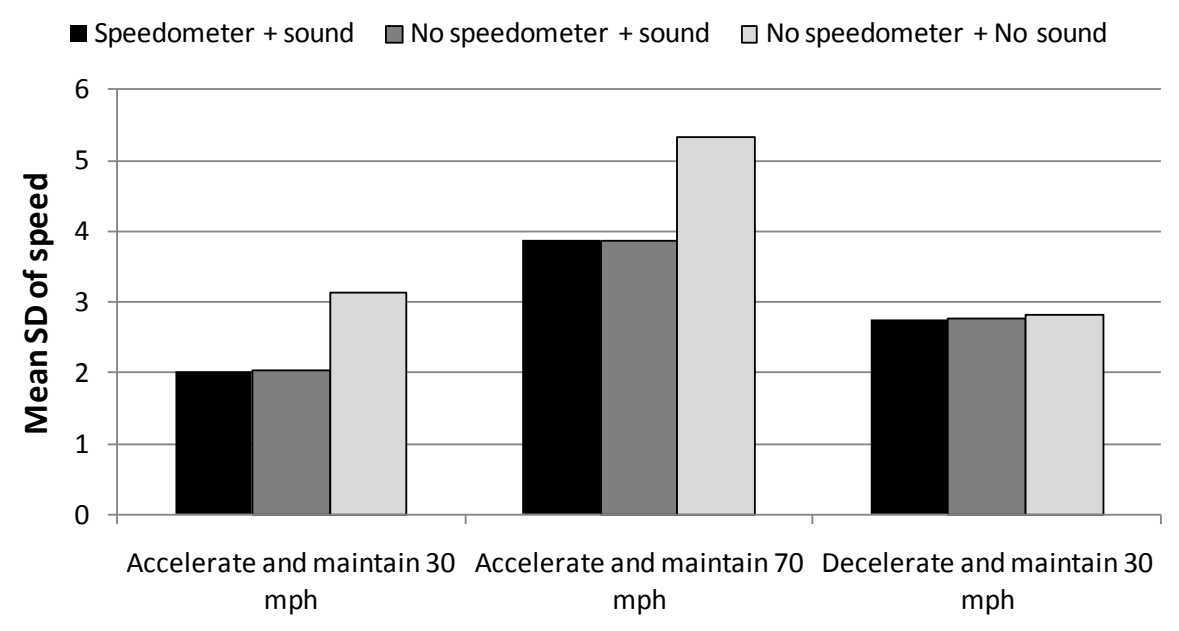

Figure 5. Mean of the standard deviation of speed for each of the three sections

There was also a significant difference in speed variation when drivers were asked to maintain speed at $30 \mathrm{mph}$ compared to $70 \mathrm{mph}$, with much more variation in the $70 \mathrm{mph}$ portions of the drive $\left(F(2,22)=15.52, p<001, \eta^{2}=.585\right)$. Finally, there was a significant interaction between speed maintenance profiles at the three sections, with significantly more variation during the 70 mph portions in section $\mathrm{C}$, where drivers did not have the assistance of a speedometer and drove in silent conditions $\left(\mathrm{F}(4,44)=3.29, \mathrm{p}<05, \eta^{2}=.230\right.$ (see

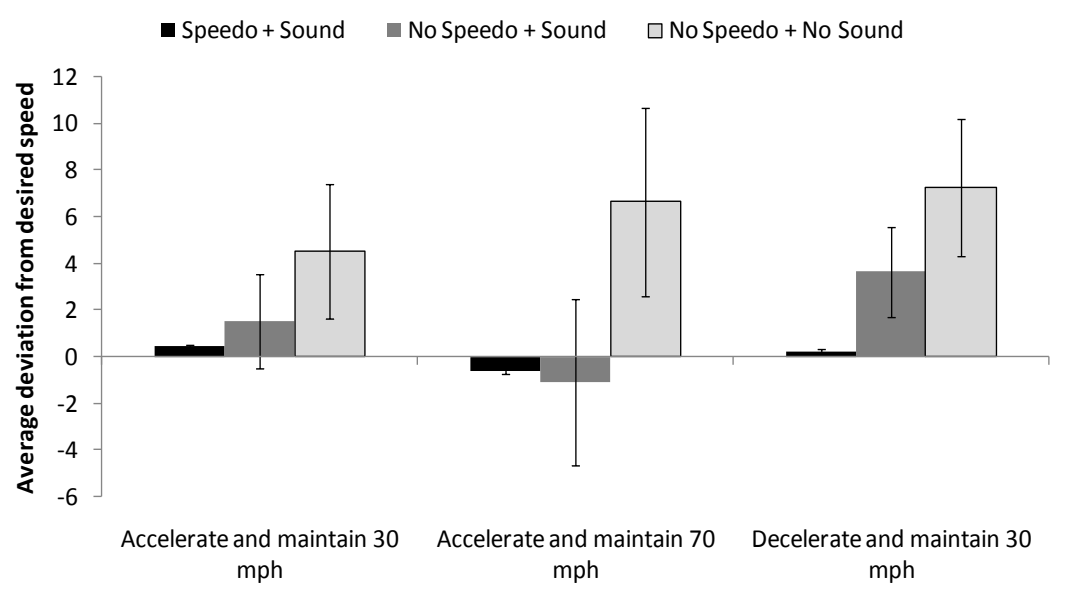

Figure 6. Deviation from the desired speed for each section and each speed profile (positive denotes driving faster than desired speed, error bars are SE)

To observe whether drivers over or underestimated their speed of travel, at each section, the average difference between required and actual speed of travel was calculated (Figure 6), and subjected to a 3 x 3 ANOVA, with the same factors as above. Drivers were found to drive faster than the required speed in the absence of engine sound $F(2,22)=4.03, p<.05, \eta^{2}=.268$ ), but there was no main effect of speed profile and no interaction between the three sections and the different speed profiles. The large errors bars in the $70 \mathrm{mph}$ section suggest that drivers found this speed most difficult to adhere to, even in the presence of engine noise. 


\section{CONCLUSIONS}

Drivers' ability to maintain the correct speed profile was much more variable in the absence of accompanying vehicle noise and this variation was found to be higher when drivers were asked to travel at higher speeds of $70 \mathrm{mph}$. Drivers were also found to travel faster than the required speed in the absence of vehicle noise, although their ability to maintain speed was generally poor at 70 miles per hour, even in the presence of auditory cues. Since the desire to manufacture and drive quieter cars is on the increase, the implications of these finding on drivers' speed perception within such vehicles is an important consideration for road safety. With the advent of more hybrid vehicles on the road, the implication on accident risk of travelling at high speeds in such vehicles must also be taken into consideration.

\section{REFERENCES}

EU, Imagine Project (2005). Harmonoise Traffic Model.See http://www.imagine-project.org/.

Evans, L. (1970). Speed estimation from a moving automobile. Ergonomics, 13, 219-230.

Gibson, J.J (1950). The Perception of the Visual World, Houghton Mifflin.

Hidaka S, Manaka, Y., Teramoto, W., Sugita, Y., Miyauchi, R., Gyoba, J., Suzuki Y, Iwaya Y. (2009). Alternation of sound location induces visual motion perception of a static object. PLoS One, 4(12)

Horswill, M. S, \& McKenna, F. P. (1999). The development, validation, and application of a video-based technique for measuring an everyday risk-taking behaviour: drivers' speed choice. Journal of Applied Psychology, 84 , 977- 985

Horswill, M.S. \& Plooy, A.M. Auditory feedback influences perceived driving speeds. Perception advance online publication. Available from http://www.perceptionweb.com/perception/fulltext/ pforth/p5736.pdf

Kemeny, A. and Panerai, F. (2003) Evaluating perception in driving simulation experiments. Trends in Cognitive Science., 7(1):31-376

Manser, M.P.\& Hancock, P.A. (2007). The influence of perceptual speed regulation on speed perception, choice, and control: Tunnel wall characteristics and influences. Accident Analysis \& Prevention, 39, 69-78.

McLane R. C.,\& Wierwille, W. W, (1975). The influence of motion and audio cues on driver performance in an automobile simulator. Human Factors, 17, 488- 501.

McGurk, H. \& MacDonald, J. (1976). Hearing lips and seeing voices. Nature 264: 746-748.

Owsley, C. \& McGwin, G. (2010). Vision and driving. Vision Research, 50 (23), 2348-2361.

Sekuler, Sekuler \& Lau (1997). Nature, 385, 308.

Shams, L., Kamitani, Y. \& Shimojo, S. (2000). What you see is what you hear. Nature, 408, 788

Sivak M, (1996). The information that drivers use: is it indeed 90\% visual? Perception, 25, 1081- 1089.

WHO Guidelines for Community Noise (2000): Berglund, B., Lindvall, T., Schwela, D.H. Guidelines for Community Noise, World Health Organisation, Geneva, 2000, p.XII. 